\title{
Calidad de vida en pacientes portadores de cardiopatías congénitas
}

\author{
María Elisa Castillo, Lida Toro R, Pamela Zelada P, Fernando Herrera L, Rodolfo Garay V, \\ Alex Alcántara P, Myriam Ferreiro C, Daniela Agusti (EU). \\ Cardiología Infantil, Hospital Dr. Sótero del Río, SSMSO, Santiago de Chile \\ Recibido el 9 de Noviembre de 2009, Aceptado el 10 de Marzo de 2010
}

\section{Resumen}

Rev Chil Cardiol 2010; 29: 57-67

Antecedentes: La corrección de las Cardiopatías Congénitas (CC) tiene como fin mejorar la calidad de vida de los pacientes portadores de ellas, pero no existen en nuestro medio estudios sobre ésta, ni su comparación con niños sanos.

Objetivos: Estudiar la calidad de vida de los CC, objetivando tres áreas, en tres grupos de pacientes de complejidad diferente, y compararlos con un grupo sano.

Método: Se escogieron 4 grupos de niños del área sur oriente de Santiago; 3 grupos de CC: comunicación interventricular (CIV), Tetralogía de Fallot (T4F), Ventrículo único (VU), y un grupo de niños sanos (S). Se sometieron a encuesta de calidad de vida ellos y sus padres, se evaluaron tres áreas especificas; actividad escolar, actividades cotidianas (actividad física y generales) y vida familiar. Se objetivó capacidad física con Test de Esfuerzo (TE), con protocolo de Bruce modificado, en todos. El análisis estadístico incluyó análisis de varianza y chi cuadrado.

Resultados: Se seleccionaron 65 niños, 12 con CIV, 18 con T4F, 15 con VU, y 20 sanos. Treinta y cuatro de ellos son hombres (52\%). En escolaridad no existen diferencias significativas en edad/curso, promedio de notas (5.7), repetición (24.8\%), en percepción de notas y rendimiento escolar los pacientes con VU tanto ellos como sus padres perciben peor rendimiento que sus pares. Con relación a actividades físicas generales el TE reveló concordancia con percepción cualitativa y diferencias de rendimientos de acuerdo a la gravedad de la patología, estadísticamente significativa sólo VU en esfuerzos mayores (S: 11:75min CIV: 12:2min, T4F:10min y VU: 7:3min). Con relación a actividades cotidianas, no perciben limitación de la vida diaria en forma significativa, excepto en algunas actividades, los pacientes con VU. Los padres de niños con CC tienen percepción de mayor limitación. Con relación a la vida familiar no hay diferencias significativas entre los 4 grupos.

Conclusiones: En general la percepción de calidad de vida de los pacientes CC no difiere en forma importante del grupo sano, existiendo algunas limitaciones en la actividad física y en algunas actividades cotidianas propias de la mayor complejidad de la cardiopatía, que implica necesidad de medicamentos, mayor número de controles, exámenes, etc. Llama la atención que el grupo sano, que debiera ser nuestro parámetro basal se calificó como limitado en actividad física y medianamente contento con la vida, lo que demuestra que nuestro concepto médico de "salud" no se correlaciona con la percepción de salud de la población general.

Correspondencia: María Elisa Castillo N.

Jefa Cardiología Infantil

Complejo Asistencial Dr. Sótero del Río

Profesor Asistente Adjunto Pontificie Universidad Católica

Concha y Toro 3456 - Puente Alto - Provincia Cordillera - Chile

Fono/Fax: (56-2) 2890419

Correo Electrónico: mecastillon@gmail.com 
M. E. Castillo, L. Toro, P. Zelada, F. Herrera, R. Garay, A.Alcántara, et al.

\section{Quality of life in patients with Congenital Heart Disease}

Background: Surgical correction of congenital heart diseases (CHD) intends to improve quality of life (QL) in affected patients. In Chile this aspect has not been objectively evaluated, especially through comparison with normal children.

Aim: to compare the quality of life in three groups of CHD patients with that of normal controls. Three aspects of QL were evaluated.

Methods: three groups of patients with CHD (Ventricular septal defect, Tetralogy of Fallot, Single Ventricle) were compared to a group of healthy children from the south east area of Santiago. A standard QL questionnaire was used to evaluate school performance, physical and general daily activities and family life. A treadmill test with a modified Bruce protocol was used to evaluate physical capacity.

Results: There were 12 patients in the VSD, 12 in the Fallot, 15 in the Single Ventricle and 20 in the normal groups. $52 \%$ were males. Age at each school level, school performance (grades and failure rates) were similar across groups except for a lower performance in patients with Single Ventricle. Physical capacity (duration of stress test) was lower in patients with Single Ventricle (7.3 min average) compared to normal (11.75min), VSD (12.2min) and Tetralogy of Fallot (10.0 min). The results of these test correlated with subjective performance of physical capacity. Similarly, patients with Single Ventricle perceived a greater limitation for daily activities (60\%) compared to VSD (100\%), Fallot's (89\%) and healthy controls (89\%). In general, parents of CHD patients perceived a greater limitation compared to their children. Family life was not different among groups.

Conclusions: The perception of QL in these patients with corrected CHD did not differ compared to healthy controls. Objective evaluation showed a lower physical capacity in patients with a more severe type of CHD.

Key words: Quality of life, Congenital Heart Disease

\section{Introducción}

El seguimiento de los CC históricamente ha sido desde la perspectiva del cardiólogo y/o cardiocirujano, y raramente desde la perspectiva del paciente y su familia ${ }^{1}$. En nuestro medio, salvo un trabajo, en que toca tangencialmente el punto de vista del paciente, no existen estudios de calidad de vida de CC desde la realidad del paciente. La perspectiva de los pacientes y sus intereses son frecuentemente diferentes a los intereses médicos e incluyen limitaciones del estilo de vida (restricción de actividad física, visitas periódicas al doctor, medicamentos, etc.), imagen de sí mismo (cicatriz de esternotomía, caja de marcapaso, etc.) y preocupación en la adolescencia de su salud futura, de la fertilidad en cardiópatas mujeres, fuentes laborales y aseguradoras de salud que los acojan. Creemos que estudios a largo plazo de la realidad de estos pacientes son de importancia vital, pues el fin último de la medicina debe ser el paciente con salud integral a largo plazo.
Estudios de calidad de vida con instrumentos validados son escasos y se han desarrollado en adultos y muestran que la evaluación física y la función motora gruesa se encuentran bajo los percentiles de la población sana, y en ellos el score de capacidad funcional de la NYHA presenta una buena correlación, pero en niños no existen estudios que relacionen salud con calidad de vida ${ }^{11}$.

Nuestro centro tiene resolución integral de los CC más complejos desde el año 1991, por lo que la mayoría de nuestros pacientes complejos están en edad escolar, y esperamos en años siguientes ampliar nuestro estudio a más patologías y adentrarnos en el período del adulto joven.

El objetivo de este trabajo es estudiar la calidad de vida de pacientes portadores de $\mathrm{CC}$ desde el punto de vista del paciente y de su familia, objetivando 3 áreas especificas: actividad escolar, actividad cotidiana (generales y físicas) e interacciones familiares, en tres 
grupos de CC con complejidad diferente, y compararla con un grupo control de niños sanos.

\section{Material y Métodos}

Tomando la base de datos de los pacientes consultantes al servicio de cardiología infantil del hospital Sótero del Río, se escogieron al azar 4 grupos de niños del área sur oriente de Santiago; 3 grupos portadores de CC de diferente complejidad: comunicación interventricular (CIV), T.Fallot (T4F), Ventrículo único (VU) y un grupo sano (S), se sometieron a encuestas de calidad de vida de salud, (*) separada en tres áreas: actividad escolar, actividades cotidianas, y vida familiar, niños y padres por separado. Al ítem de escolaridad se agregó edad-curso y promedio de notas para objetivarla mejor. En actividad física se realizó además Test de esfuerzo (TE), con protocolo de Bruce modificado. Se analizaron las encuestas y se compararon de acuerdo a las 3 áreas específicas evaluadas. Las encuestas de calidad de vida fueron tomadas de encuestas utilizadas en San Diego, EEUU, y traducidas al español y validadas para la población del área Sur-Oriente en estudio. El análisis estadístico incluyó análisis de varianza y chi cuadrado.

\section{Resultados}

Se seleccionan 65 niños en total de los cuales 12 tienen CIV con edad promedio de 12.9 años, 18 presentan T4F con edad promedio de 11 años, 15 tienen VU con edad promedio de 11.3 años, y 20 son sanos con edad promedio de 12 años. Treinta y cuatro de ellos son hombres $(52 \%)$. Los pacientes con CIV tuvieron 1 cirugía y se encuentran sin patología residual, los con T4F tuvieron 1.3 cirugía /niño y tienen patología residual, los con VU tuvieron 3.5 cirugía/ niño y tienen patología residual. (tabla1).

Tabla 1: Características generales

\begin{tabular}{lccccc}
\hline & CIV & T4Fallot & VU & Sano & TOTAL \\
\hline $\mathrm{N}^{\circ}$ & 12 & 18 & 15 & 20 & 65 \\
Edad promedio & 12,9 & 11 & 11,3 & 12 & 11,8 \\
Hombres & $8 / 4$ & $10 / 8$ & $7 / 8$ & $9 / 11$ & $34 / 11$ \\
Mujeres & 1 & 1,3 & 3,5 & & \\
$\mathrm{~N}^{\circ}$ cirugías / niño & & & & \\
\hline
\end{tabular}

En escolaridad no existen diferencias significativas en edad/curso, promedio de notas (5.7), cursos repetidos (24.8\%); lo mismo en percepción de notas y rendimiento escolar. Los Ventrículos Únicos tanto pacientes como padres perciben peor rendimiento que el curso, pero no es estadísticamente significativo. (Tabla2)

Tabla 2: Escolaridad

\begin{tabular}{|c|c|c|c|c|}
\hline Escolaridad & Normal & CIV & $\mathrm{T} 4 \mathrm{~F}$ & VU \\
\hline edad & 12 & 13 & 11 & 11 \\
\hline curso & 6 Básico & 7 Básico & 5 Básico & 6 Básico \\
\hline Promedio notas & 5,9 & $5,7(p>0,05)$ & $5,8(p>0,05)$ & $5,6(p>0,05)$ \\
\hline Paciente Muy & & & & \\
\hline contento notas & $42 \%$ & $33 \%(p>0,05)$ & $22 \%(p>0,05)$ & $13 \%(p=0,05)$ \\
\hline Padre & & & & \\
\hline contento & $31 \%$ & $25 \%(p>0,05)$ & $11 \%(p>0,05)$ & $20 \%(p>0,05)$ \\
\hline
\end{tabular}


M. E. Castillo, L. Toro, P. Zelada, F. Herrera, R. Garay, A.Alcántara, et al.

La actividad física fue evaluada cualitativa y cuantitativamente. Para objetivar la limitación física se aplicó Test de esfuerzo cuyo resultado se resume en Tabla 4 La limitación en actividades físicas fue catalogada en limitación de: mayor, mediana y leve esfuerzo. Fue considerado esfuerzo mayor: realizar algún deporte competitivo, jugar fútbol. Esfuerzo mediano: andar en bicicleta, en patines, bailar. Esfuerzos leves: agacharse, caminar. Sólo existe diferencia estadísticamente significativa en el grupo de VU frente a esfuerzos mayores y medianos. Los resultados se resumen en la Tabla 3 . Los padres en general tienen una percepción de mayor limitación en la actividad física que los niños, pero no es estadísticamente significativa (en la tabla 3 sector derecho se compara estadísticamente percepción pacientes-padres).

Tabla 3: Limitación en Actividad física Pacientes

Padres

\begin{tabular}{|c|c|c|c|c|c|c|c|c|}
\hline $\begin{array}{l}\text { Grado } \\
\text { Limitación }\end{array}$ & CIV & T4Fallot & VU & Sano & CIV & T4F & VU & $S$ \\
\hline $\begin{array}{l}\text { Esfuerzo } \\
\text { mayor }\end{array}$ & $17 \%$ & $37 \%(p>0,001)$ & $80 \%(p<0,01)$ & $26,4 \%$ & $34 \%(p>0,01)$ & $47 \%(p>0,05)$ & $80 \%$ & $21 \%(p>0,05)$ \\
\hline $\begin{array}{l}\text { Esfuerzo } \\
\text { Mediano }\end{array}$ & $8,4 \%$ & $34 \%(p>0,01)$ & $60 \%(p<0,05)$ & $26,4 \%$ & $34 \% p=0,051$ & $34 \%$ & $73,4 \% p>0,05$ & $10,5 \%(p>0,05)$ \\
\hline $\begin{array}{l}\text { Esfuerzo } 1 \\
\text { Leve }\end{array}$ & $17 \%(p>0,05)$ & $28 \%(p>0,01)$ & $27 \%(p>0,01)$ & $15,8 \%$ & $8,3 \%(p>0,01)$ & $34 \%(p>0,05)$ & $61 \%(p>0,01)$ & $10,5 \%(p>0,05)$ \\
\hline
\end{tabular}

Tabla 4: Test de Esfuerzo

\begin{tabular}{lcccc}
\hline Test esfuerzo & CIV & T4Fallot & VU & Sano \\
\hline Minutos totales & 12,2 min & 10 min & 7,3 min & 11,7 min \\
Estapas completas & $4 \mathrm{Et}(p>0,01)$ & $3 \mathrm{Et}(p>0,01)$ & $2 \mathrm{Et}(p<0,05)$ & $3 \mathrm{Et}$ \\
\hline
\end{tabular}

Como segundo ítem de la encuesta se evaluaron las interacciones familiares con diferentes preguntas Se les pidió que calificaran la vida familiar en general, con los adjetivos de muy buena, buena, regular o mala (Tabla 5).

Tabla 5: Calificación de la Vida Familiar

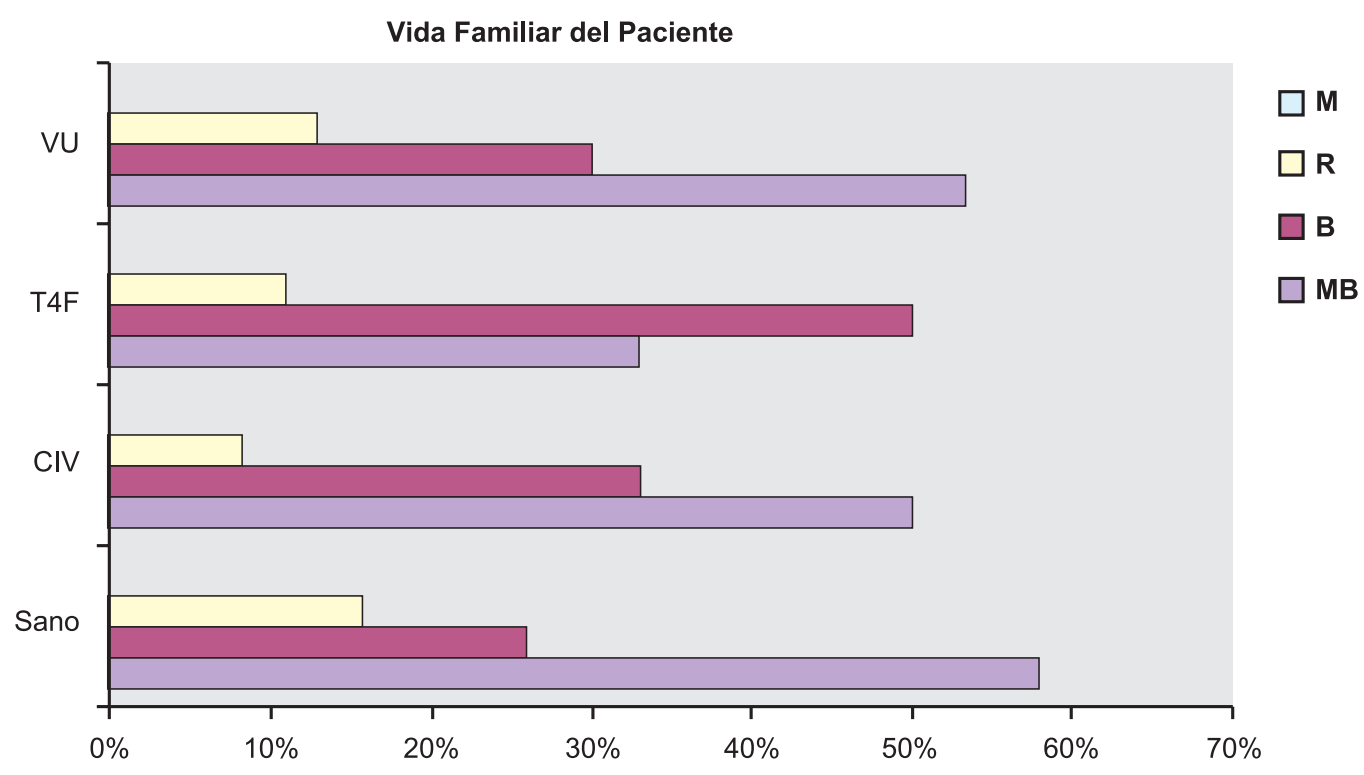

MB: muy buena T4F p>0,01, B: buena, VU p>0,01 R: regular, CIV p>0,01 M:mala 
Se les pidió que evaluaran la peor y la mejor relación con el resto de los integrantes de la familia, que se resume en la tabla 6 . No se encontraron diferencias significativas entre los grupos.

Tabla 6: Relaciones con los integrantes de la familia

\begin{tabular}{lllccc}
\hline & Sano & CIV & T4F & VU \\
\hline \multirow{3}{*}{ Mejor relac. } & Mamá & $63 \%$ & $42 \%(p<0.05)$ & $33 \%(p<0,01)$ & $47 \%(p>0.05)$ \\
& Papá & $26 \%$ & $25 \%$ & $22 \%$ & $47 \%(p>0,01)$ \\
& Hermano & $10 \%$ & $33 \%(p>0,05)$ & $33 \%(p>0,05)$ & $7 \%(p>0.01)$ \\
Peor relac. & & & & $16,7 \%$ & $13 \%$ \\
& Papá & $15 \%$ & $25 \%(p>0.01)$ & $9 \%$ & $0 \%$ \\
& Mamá & $15 \%$ & $8 \%(p>0,01)$ & $61(p>0,01)$ & $47 \%(p>0,01)$ \\
\hline
\end{tabular}

Se les preguntó a las madres por el grado de angustia que les provoca la salud de su hijo (Tabla 7), y por el grado de angustia que les provoca la salud emocional de su hijo (Tabla 8). No se encontraron diferencias significativas en relación a angustia por salud, como tampoco en angustia en comportamiento, excepto en el grupo con CIV donde la diferencia fue límite, aunque no significativa.

Tabla 7: Angustia materna por la salud del hijo

\section{Angustia por Salud del Hijo}
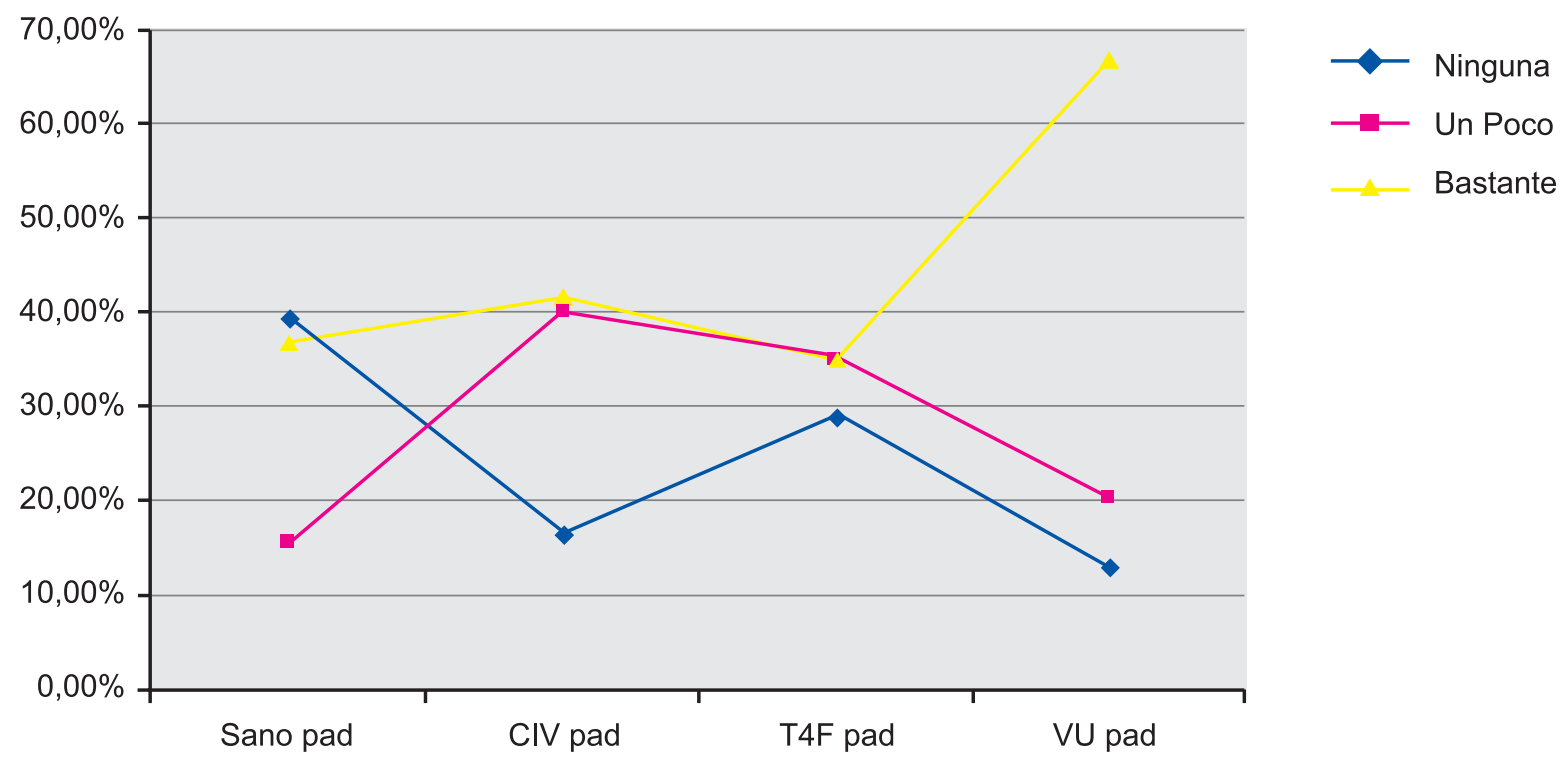

Bastante: VU $p>0,01$ Ninguna VU $p>0,01$ 
M. E. Castillo, L. Toro, P. Zelada, F. Herrera, R. Garay, A.Alcántara, et al.

Tabla 8: Angustia materna por la salud emocional del hijo

Angustia por Salud Emocional del Hijo

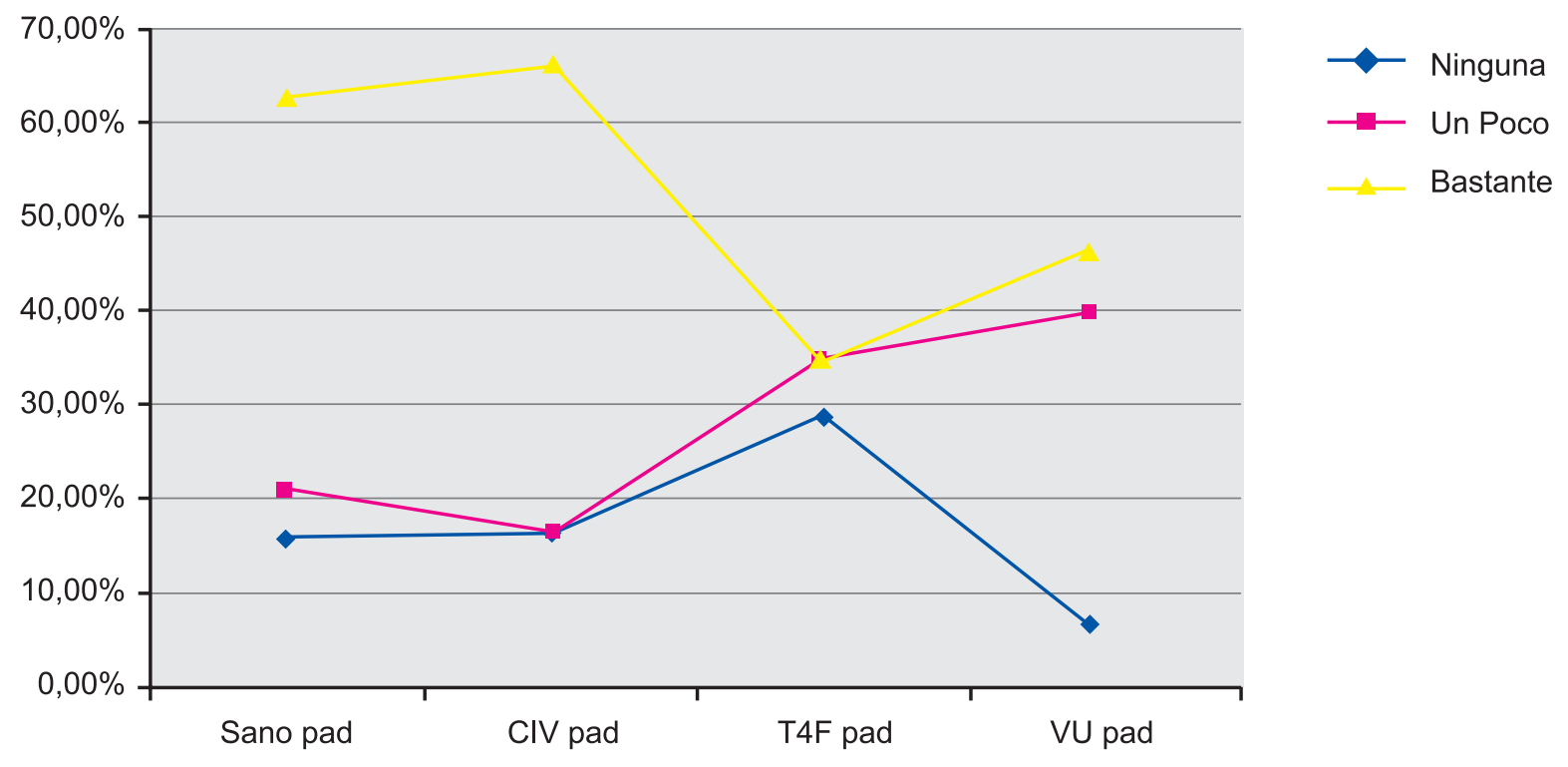

Bastante: CIV $p=0,051$, Ninguna VU $p>0,05$

Para evaluar la percepción de los padres con relación a sí la enfermedad de su hijo ha afectado la vida familiar se les preguntó si tenían mayor dedicación de tiempo al hijo que al resto de la familia (Tabla 9, VU y T4F con variación significativa), si se preocupaban más que otros padres (Tabla 10, el grupo de padres de pacientes con VU con diferencia significativa), si existía limitación de actividades familiares por la salud del hijo (Tabla 11, sin diferencias significativas), o limitación de actividades familiares por el comportamiento del hijo (Tabla 12, sin diferencias significativas) y finalmente si pensaban que existía alteración de la relación de pareja por la salud del hijo (Tabla 13, sólo existía diferencia en grupo de pacientes con VU). Todo esto con el fin de caracterizar mejor el impacto intrafamiliar que provoca tener un hijo cardiópata, y la percepción de cada uno de sus integrantes.

Como último ítem dentro de la encuesta, se evaluaron actividades de la vida diaria.

Se les preguntó con relación a actividades de la vida cotidiana, de su sensación de limitación por su enfermedad, de sus amistades y de su percepción general de la vida, (Tabla 14, no hubo diferencias significativas excepto grupo de $\mathrm{VU}$ en relación a diferencia con los pares). Las encuestas de los padres de niños con CC mostraron que éstos tienen percepción de mayor limitación que sus hijos, siendo significativa sólo pacientes-padres con CIV. 
Tabla 9: Mayor tiempo dedicado al hijo

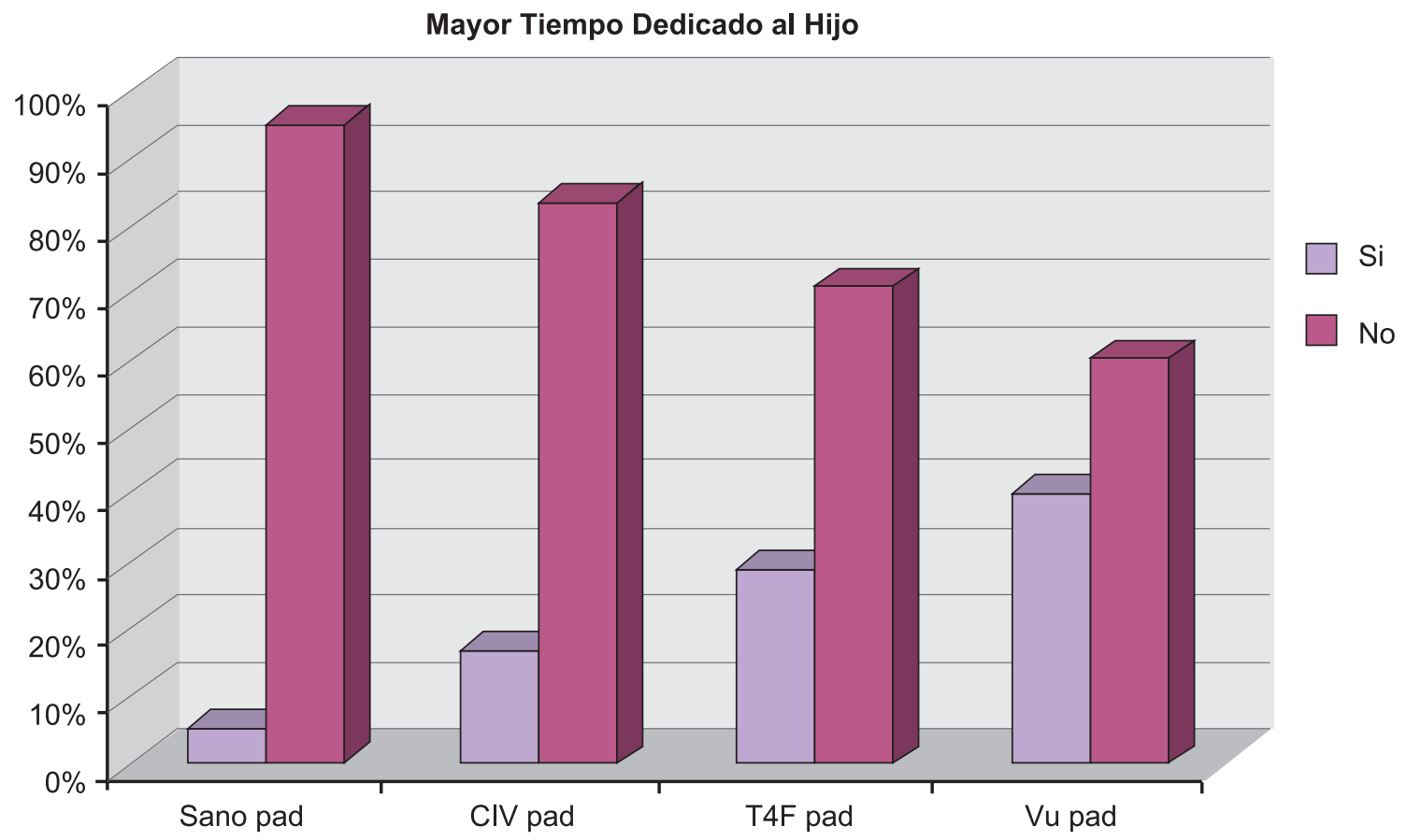

SI: VU $p<0,01 ;$ T4F $p<0,05 ;$ CIV $p>0,01$

Tabla 10: Mayor preocupación que otros Padres

Mayor preocupación que otros Padres

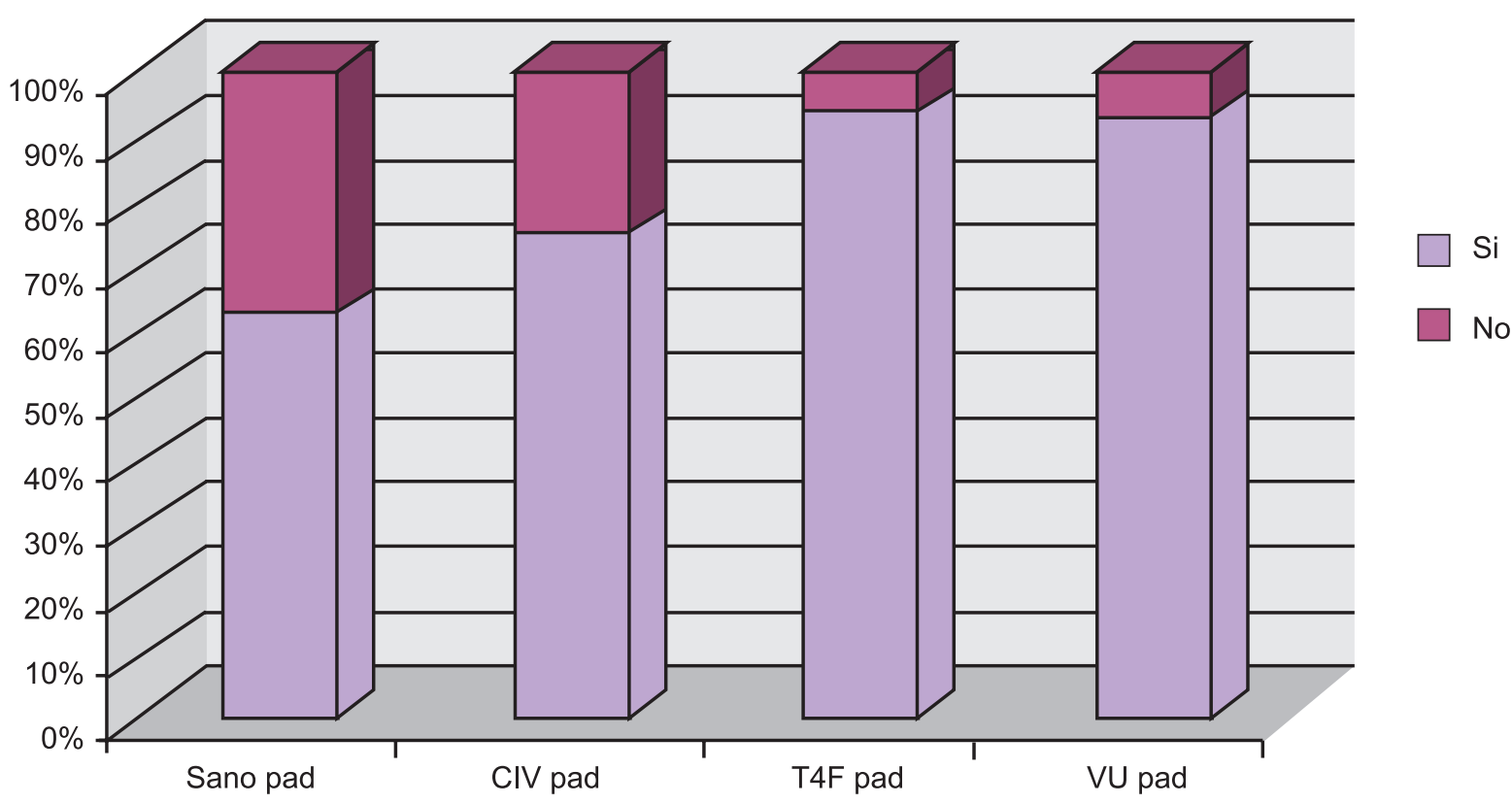

NO: VU $p<0,05 ;$ T4F $p<0,05 ;$ CIV $p>0,05$ 
M. E. Castillo, L. Toro, P. Zelada, F. Herrera, R. Garay, A.Alcántara, et al.

Tabla 11: Limitación de actividades familiares por salud del hijo

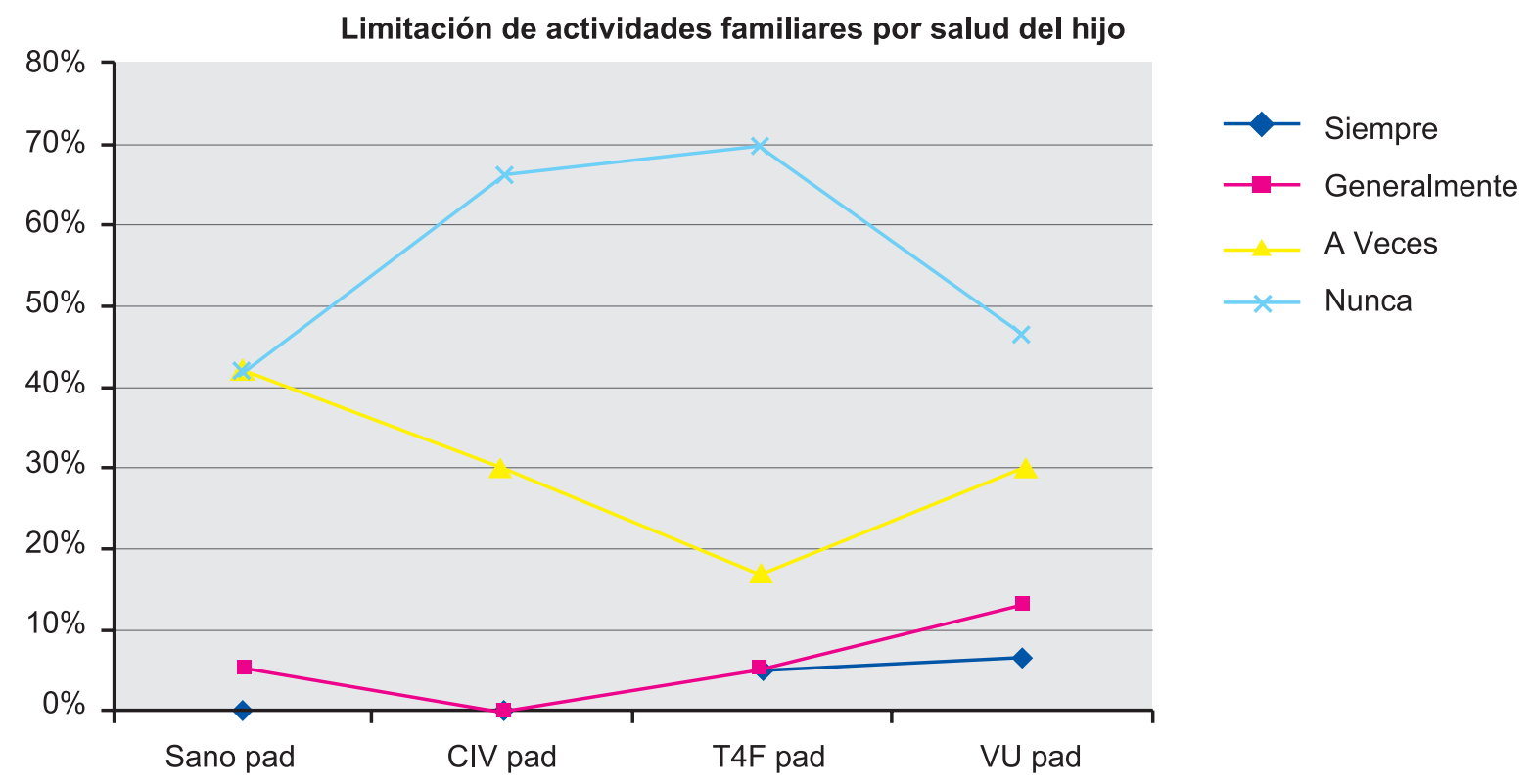

Bastante: VU $p>0,01$ Ninguna VU $p>0,01$

Tabla 12: Limitación de Actividades Familiares por comportamiento del hijo

\section{Limitación de Actividades Familiares por comportamiento}

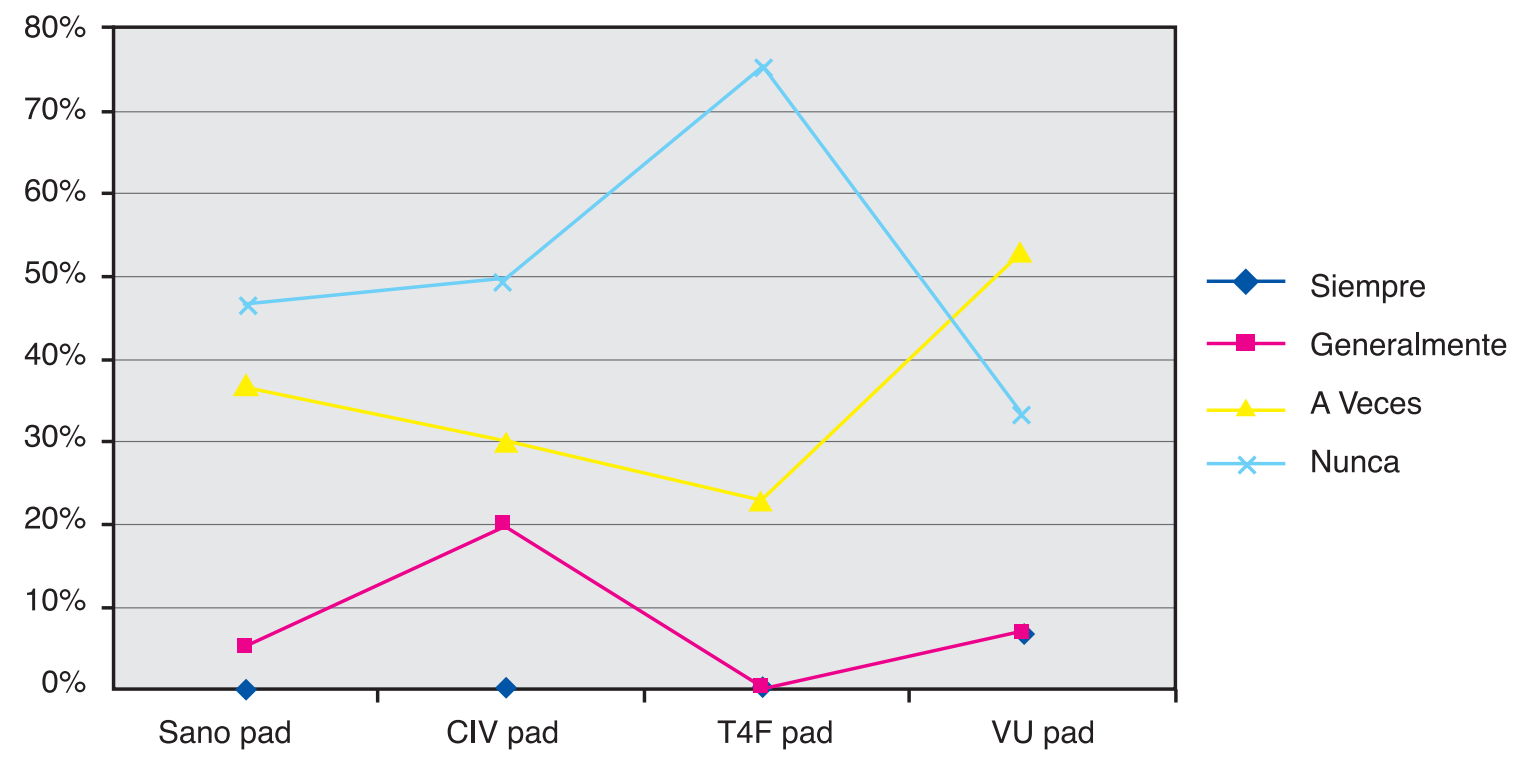

Nunca T4F $p>0,05$; Generalmente CIV $p>0.05$ 
Tabla 13. Alteración de la Relación de pareja por la salud del hijo

\section{Alteración Relación de Pareja por Salud del Hijo}

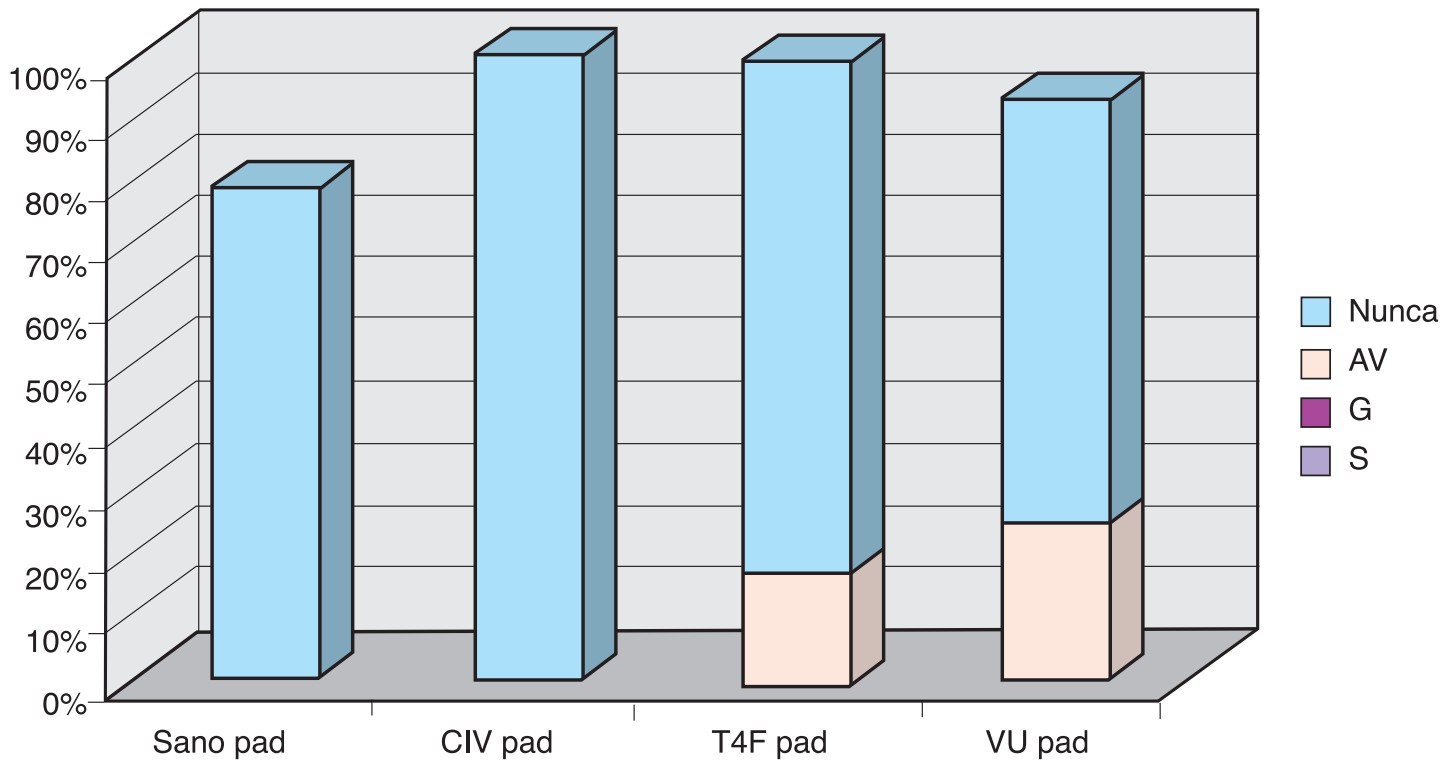

Nunca VU $p<0,05 ;$ T4F $p>0,01$

Tabla 14: Actividades cotidianas

\begin{tabular}{|c|c|c|c|c|c|c|c|c|}
\hline \multirow[b]{2}{*}{$\begin{array}{l}\text { Actividades } \\
\text { Cotidianas }\end{array}$} & \multicolumn{3}{|c|}{ Pacientes } & \multicolumn{5}{|c|}{ Padres } \\
\hline & CIV & T4Fallot & VU & Sano & CIV & T4F & VU & $\mathbf{S}$ \\
\hline Salud no limita & $\begin{array}{c}100 \% \\
(P>0,05)\end{array}$ & $\begin{array}{c}89 \% \\
(P>0,05)\end{array}$ & $\begin{array}{c}60 \% \\
(P>0,01)\end{array}$ & $89 \%$ & $\begin{array}{c}60 \% \\
(P<0,01)\end{array}$ & $\begin{array}{c}71 \% \\
(P>0,05)\end{array}$ & $60 \%$ & $\begin{array}{c}95 \% \\
(P>0.05)\end{array}$ \\
\hline $\begin{array}{l}\text { Diferente a sus } \\
\text { pares }\end{array}$ & $\begin{array}{c}16,7 \% \\
(p>0,05)\end{array}$ & $\begin{array}{c}22 \% \\
(p>0.05)\end{array}$ & $\begin{array}{c}66,6 \% \\
(p<0,01)\end{array}$ & $5 \%$ & & & & \\
\hline $\begin{array}{l}\text { Contento con } \\
\text { la vida }\end{array}$ & $\begin{array}{c}83 \% \\
(p>0,01)\end{array}$ & $\begin{array}{c}44 \% \\
(p>0,05)\end{array}$ & $\begin{array}{c}46,6 \% \\
(p>0.05)\end{array}$ & $59 \%$ & $\begin{array}{c}16,6 \% \\
(p<0.01)\end{array}$ & $\begin{array}{c}36 \% \\
(p>0,05)\end{array}$ & $\begin{array}{c}60 \% \\
(p>0,05)\end{array}$ & $\begin{array}{c}42 \% \\
(p>0.05)\end{array}$ \\
\hline
\end{tabular}

\section{Discusión}

Desde el punto de vista del rendimiento escolar, la literatura ${ }^{2,5}$ describe alteraciones en el neurodesarrollo de pacientes portadores de VU con Coeficiente Intelectual dentro de rango normal, pero más lentos que la población general. Son descritas alteraciones de integración espacio-visual y motora-visual y de funciones cognitivas. Varios artículos describen más detalladamente estas alteraciones ${ }^{3}$, 4, 6,7. Por eso llama la atención que en nuestro trabajo no existan diferencias significativas en rendimiento escolar, promedio de notas, y edad/curso entre los pacientes con CC y el grupo sano. Además los pacientes con VU perciben su rendimiento peor que el de sus pares, teniendo promedio de notas muy similar al grupo de sanos, que se encuentran conformes con sus notas. 
En actividades físicas; La capacidad de realizar ejercicios después de cardiocirugía en CC depende de sí la cirugía ha sido correctora o paliativa y de la presencia de defectos residuales (shunt residuales, disfunciones valvulares, presencia de arritmias significativas, disfunciones miocárdicas, etc). Nuestro estudio presenta 3 grupos diferentes; sin patología residual (CIV), con patología residual (T4F) y con cirugía paliativa (VU). La literatura muestra que la capacidad física se encuentra disminuida en diversos grados dependiendo de la complejidad de la $\mathrm{CC}^{8,9,10}$ lo que encontramos también en nuestro estudio, pero siendo significativa sólo en el grupo más complejo. Los estudios muestran también que la limitación física es percibida como menor por los pacientes a la objetivada en Test específicos, lo mismo muestra nuestro estudio.

Llama la atención en nuestro estudio que del grupo de niños sanos un $16 \%$ se considere limitado ante esfuerzos mínimos y un $26.4 \%$ lo perciba con esfuerzos medianos y grandes (¿comprensión de lectura de la encuesta?). Llama la atención que los padres de este grupo tengan una percepción semejante a los niños. Este mismo grupo sólo alcanza estadio 3 del Test de esfuerzo, indudablemente el sedentarismo de nuestra población es un hecho alarmante, siendo niños con un promedio de edad de 11 años, edad de gran actividad física en general.

En la vida familiar, desde la perspectiva del paciente, prácticamente no existen diferencias significativas en los 4 grupos. Los ítems, mayor preocupación que otros padres y mayor tiempo dedicado al niño son los que muestran más diferencia significativa de los grupos más complejos con respecto al grupo sano. Es lógico que a mayor complejidad más tiempo y preocupación, pero llama la atención que padres de niños sanos tengan un $63 \%$ de mayor preocupación por su hijo sano.

Desde la perspectiva de los padres es lógico que la angustia por la salud del hijo sea mayor en los padres de niños con $\mathrm{VU}$, aunque la diferencia no es signifi- cativa. Llama la atención que los padres de niños sanos tengan mayor angustia que padres de niños con CIV, aunque no significativa, no tenemos explicación para este hallazgo, son niños sin patología alguna.

Con relación a la angustia por comportamiento del niño, la tienen más los padres de niños con CIV, límite de ser significativo y niños sanos, pero sin diferencia significativa; a diferencia de lo mostrado por la literatura ${ }^{12}$, probablemente por la edad de los niños estudiados que son todavía muy dependientes de los padres.

En el ítem limitación de actividades por la salud del niño se observa una percepción de adecuada vida familiar a pesar de la enfermedad del niño, nuevamente el grupo de padres de niños sanos tiene una percepción de mayor limitación en este ítem que padres de niños con CIV y T4F, no siendo significativa la diferencia. En la evaluación de impacto en la vida de pareja sólo existe impacto en los padres de niños más complejos, lo cual es dable esperar.

Vida cotidiana: Ante la pregunta de si la salud limita las actividades diarias, sólo los niños portadores de VU perciben estar significativamente limitados, pero esta percepción no presenta diferencia significativa con el grupo control. Los niños con T4F no se perciben limitados, probablemente por su edad (sin compromiso sintomático de su patología residual). Llama la atención que los niños sanos se perciban igualmente limitados que los con T4F. Los padres de los 4 grupos, en general tienen una percepción de mayor limitación que los pacientes, sólo es estadísticamente diferente en el grupo padres-pacientes con CIV. Los padres de niños sanos tienen similar percepción que sus hijos con relación a su limitación. Ante la constante limitación percibida por el grupo de niños sanos no tenemos respuesta.

La percepción de ser diferente al resto es proporcional a la complejidad de la cardiopatía y corresponde a lo esperado, siendo significativo sólo en el grupo de 
VU. Con relación a la satisfacción con la vida y amistades, los que muestran diferencia en estar contento y muy contentos son los niños con CIV, aunque esta diferencia no es significativa; probablemente haber tenido una patología y estar hoy en día sanos los hace tener ante la vida una actitud más positiva.
Pensamos que este estudio de calidad de vida es una primera aproximación al entendimiento del concepto de "salud integral" de este grupo de pacientes, y nos aporta puntos de vista diferentes, que probablemente debemos incluir dentro del seguimiento y evaluación de resultados de este grupo de pacientes.

\section{REFERENCIAS:}

1. AMY H. Schultz and Gil Wernovsky Late Outcomes in Patients with Surgically Treated Congenital Heart Disease Semin Cardiovasc Sur Pediatr Cardiac Surg Ann 2005; 8:145-156.

2. WERNOVSKY G, STILES KM, GAVREAU K, GENTLES TL, DUPLESSIS AJ, BELLINGER DC, et al. Cognitive development after the Fontan operation Circulation 2000; 102:883-889.

3. NEWBURGER JW, WYPIJ D, BELLINGER DC, DU PLESSIS AJ, KUBAN KC, RAPPAPORT LA, et al. Neurodevelopment status at eight years in children with dextro_transposition of the great aetrries: The BostonCirculatory Arrest Trial. J.Thorac Cardiovsac Surg 2003; 126: 1385-1396.

4. MAHLE WT, CLANCY RR, MOSS EM, GERDES M, JOBES DR, WERNOVSKY G. Neurodevelopment outcome and lifestyle assesment in school and adolescent children with hipoplastic left heart syndrome: Peatrics 2000; 105 : 1082-1089.

5. BELLINGER DC, WYPIJ D, KUBAN KC, RAPPAPORT LA, HICKEY PR, WERNOVSKY G, et al. Development and neurological status of children at 4 years after heart surgey with hypothermic circulatory arrest or low flow cardiopulmonary bypass circulation 1999; 100: 526-532.

6. MAHLE WT, WERNOVSKY G. Long-term development outcome of children with complex congenital heart disease. Clin Perinatol 2001; 28: 235-247.

7. MAJNEMER A, LIMPEROPOULOS C. Development progress of children with congenital heart defects requiring heart surgery. Semin Pediatr Neurol 1999; 6: 2-19.

8. DRISCOLL DJ, DURONGPISITKUL K. Exercise testing after the Fontan operation. Pediatric Cardiol 1999; 20: 5759.

9. WESSEL HU, PAUL MH. Exercise studies in tetralogy of Fallot: A review Pediatr Cardiol 1999; 20: 39-47.

10. HELBER $U$, BAUMANN R, SEBOLDT $H$, REINHARD U, HOFFMEISTER HM. Atrial septa defect in adults: cardiopulmonary exercise capacity before and 4 months and 10 years after defect closure. J Am Coll Cardiol 1997; 29:1345-1350.

11. MOONS P, VAN DEYK K, BUDTS W, DE GEEST S. Caliber of quality-of-life assesments in congenital heart disease: A plea for more conceptual and methodologic rigor.Arch Pediatr Adolesc Med 2004, 158: 1062-1069.

12. SCHILLINGFORD AJ, WERNOVSKY G. Academic performance and behavioral difficulties after neonatal and infant heart surgery. Pediatr Clin North Am 2004; 51 : 1625-1639.

13. LANE DA, LIP GY, MILLANE TA. Quality of life in adults with congenital heart disease. Heart 2002; 88: 71-75.

14. KAMPHUIS M, OTTENKAMP J, VLIEGEN HW, VOGELS T, ZWINDERMAN KH, KAMPHUIS RP, et al. Health related quality of life and health status in adults survivors with previously operated complex congenital heart disease. Heart 2002; 87: 356-362. 